\title{
Treponema denticola chymotrypsin-like proteinase may contribute to orodigestive carcinogenesis through immunomodulation
}

Mikko T Nieminen*,1,12, Dyah Listyarifah ${ }^{2,3,12}$, Jaana Hagström ${ }^{4,5}$, Caj Haglund ${ }^{5,6}$, Daniel Grenier ${ }^{7}$, Dan Nordström ${ }^{8}$, Veli-Jukka Uitto ${ }^{1}$, Marcela Hernandez ${ }^{9,10}$, Tülay Yucel-Lindberg ${ }^{11}$, Taina Tervahartiala ${ }^{1}$, Mari Ainola ${ }^{3}$ and Timo Sorsa ${ }^{1,11}$

${ }^{1}$ Department of Oral and Maxillofacial Diseases, University of Helsinki and Helsinki University Central Hospital, Haartmaninkatu 8 , Helsinki 00029, Finland; ${ }^{2}$ Department of Dental Biomedical Sciences, Faculty of Dentistry, Universitas Gadjah Mada, Jl. Denta Sekip Utara no 1, Yogyakarta 55281, Indonesia; ${ }^{3}$ Department of Medicine, Clinicum, University of Helsinki and Helsinki University Central Hospital, Haartmaninkatu 8, Helsinki 00029, Finland; ${ }^{4}$ Department of Pathology, Haartman Institute and HUSLab, University of Helsinki and Helsinki University Hospital, PO Box 21, Haartmaninkatu 3, 00014 Helsinki, Finland; ${ }^{5}$ Research Program Unit, Translational Cancer Biology, University of Helsinki, Helsinki, Finland; ${ }^{6}$ Department of Surgery, University of Helsinki and Helsinki University Central Hospital, PO Box 340, Helsinki 00029, Finland; ${ }^{7}$ Faculté de Médecine Dentaire, Université Laval, Quebec City, Quebec G1V OA6, Canada; ${ }^{8}$ Department of Internal Medicine and Rehabilitation, University of Helsinki and Helsinki University Central Hospital, Helsinki 00029, Finland; ' Laboratory of Periodontal Biology and Department of Oral Pathology and Medicine, Faculty of Dentistry, University of Chile, Santiago, Chile; ${ }^{10}$ Dentistry Unit, Faculty of Health Sciences, Universidad Autónoma de Chile, Santiago, Chile and ${ }^{11}$ Division of Periodontology, Department of Oral Diseases, Karolinska Institutet, Box 4064, Huddinge SE-141 04, Sweden

Background: Periodontal pathogens have been linked to oral and gastrointestinal (orodigestive) carcinogenesis. However, the exact mechanisms remain unknown. Treponema denticola $(T d)$ is associated with severe periodontitis, a chronic inflammatory disease leading to tooth loss. The anaerobic spirochete $T d$ is an invasive bacteria due to its major virulence factor chymotrypsinlike proteinase. Here we aimed to investigate the presence of Td chymotrypsin-like proteinase (Td-CTLP) in major orodigestive tumours and to elucidate potential mechanisms for $\mathrm{Td}$ to contribute to carcinogenesis.

Methods: The presence of Td-CTLP within orodigestive tumour tissues was examined using immunohistochemistry. Oral, tonsillar, and oesophageal squamous cell carcinomas, alongside gastric, pancreatic, and colon adenocarcinomas were stained with a Td-CTLP-specific antibody. Gingival tissue from periodontitis patients served as positive controls. SDS-PAGE and immunoblot were used to analyse the immumodulatory activity of Td-CTLP in vitro.

Results: Td-CTLP was present in majority of orodigestive tumour samples. Td-CTLP was found to convert pro MMP-8 and -9 into their active forms. In addition, Td-CTLP was able to degrade the proteinase inhibitors TIMP-1, TIMP-2, and $\alpha$ - 1 -antichymotrypsin, as well as complement C1q.

Conclusions: Because of its presence within tumours and regulatory activity on proteins critical for the regulation of tumour microenvironment and inflammation, the Td-CTLP may contribute to orodigestive carcinogenesis.

\footnotetext{
*Correspondence: Dr M Nieminen; E-mail: mikko.t.nieminen@helsinki.fi

${ }^{12}$ These authors contributed equally to this work.
}

Received 7 July 2017; revised 16 October 2017; accepted 18 October 2017; published online 16 November 2017

(C) 2018 Cancer Research UK. All rights reserved 0007-0920/18 
Over the past few decades, a paradigm shift has been made in understanding the key role of the microbiome in health and disease. Discovery of the causative role of Helicobacter pylori in gastric cancer has been one of the driving factors in this area. It has been estimated that $20 \%$ of all cancers have a microbial aetiology (Parkin, 2006). Accumulating evidence has shown that microbes have a more potent role in gastrointestinal (GI) carcinogenesis than previously thought. Various members of the oral microbiota have been reported to be linked to cancers of the GI tract (Fitzpatrick \& Katz, 2010; Ahn et al, 2012a, b).

In the oral microbiome, the so-called periodontopathogens, including Fusobacterium nucleatum (Fn), Porphyromonas gingivalis $(P g)$, Treponema denticola (Td), Aggregatibacter actinomycetemcomitans $(\mathrm{Aa})$, and Tannerella forsythia (Tf) have been receiving increasing interest in the context of cancer aetiology. Periodontal microbes have a major role in the pathogenesis of periodontitis, chronic inflammatory disease that causes destruction of the tooth-supporting tissue and ultimately tooth loss. Novel epidemiological studies have linked periodontal pathogens to pancreatic and oral cancers (Ahn et al, 2012a; Michaud, 2013). Also, it has been reported that $F n$ can promote colorectal carcinogenesis (Rubinstein et al, 2013). Both in vitro and in vivo studies have suggested that $F n$ and $P g$ participate in oral carcinogenesis (Groeger et al, 2011; Gallimidi et al, 2015). Oral bacteria may contribute to carcinogenesis via different mechanisms such as inhibition of apoptosis, activation of cell proliferation, promotion of cellular invasion, induction of chronic inflammation, and production of carcinogens (Gallimidi et al, 2015). Bacterial pathogens have developed various mechanisms that can promote their ability to colonise the GI tract: strong adhesive properties; immune evasion; disruption of epithelial integrity; and intracellular survival (Ribet \& Cossart, 2015).

Within the oral microbiome, $T d$ is the most well-characterised and frequently isolated spirochete (Dashper et al, 2011). In addition to its association with periodontal disease, $T d$ has been reported to contribute to the formation of dental abscesses and root canal infections. It is a highly invasive and motile organism, and an obligate anaerobe. One of the key virulence factors of $T d$ is a cell surface-bound chymotrypsin-like proteinase (CTLP), also known as dentilisin, which has a plethora of functions. It can degrade multiple host proteins, hydrolyse bioactive peptides, contribute to $T d$ penetration into the epithelium, and enhance integration of $T d$ into biofilm communities (Grenier et al, 1990). In addition, Td-CTLP can modulate immunity and inflammation, and drive apoptosis in various cell types (Uitto et al, 1995; Cogoni et al, 2012; Fenno, 2012). However, results reporting the association of $T d$ with malignancies are very limited and only one study has reported the presence of $T d$ in oesophageal tumours (Narikiyo et al, 2004).

Host-derived matrix metalloproteinases (MMPs) have a critical role in inflammatory processes and are capable of degrading almost all extracellular proteins. MMPs are secreted by inflammatory cells, in concert with endothelial, epithelial, and smooth muscle cells. They facilitate leukocyte navigation through the extracellular matrix (ECM) and modify immune responses by processing non-matrix bioactive substrates (Salminen et al, 2015). The key regulators of MMP activity are tissue inhibitors of MMPs (TIMPs). There is a broad evidence highlighting the importance of MMPs and TIMPs in the regulation of tumour microenvironment and in the metastasis of GI cancers (Jiang et al, 2002; Verma et al, 2014).

In addition, other protease inhibitors are linked to carcinogenesis. Alpha-1-antichymotrypsin $(\alpha-1-\mathrm{AC})$ is an acute-phase protein belonging to the serpin superfamily of protease inhibitors with activity against various proteases such as neutrophil elastase and cathepsin G. It has critical functions in tissue homeostasis and its deficiency leads to chronic inflammation, which is associated with several types of cancer, including gastric and colorectal cancers
(Kittas et al, 1982; Karashima et al, 1990; Sun \& Yang, 2004). The complement system has been known as the pivotal arm of the innate immunity. In addition, being able to activate of the classical pathway, complement $\mathrm{Clq}$ is known to be expressed in several cancer tissues, including colorectal and pancreatic cancers (Bulla et al, 2016).

In this study, we examined the presence of Td-CTLP in major oral and GI (orodigestive) tumours in vivo using immunohistochemistry. In addition, we also investigated the ability of Td-CTLP to modulate immunomodulatory proteins, including MMPs, TIMPs, $\alpha-1-\mathrm{AC}$, and C1q in vitro. The association of Td-CTLP with orodigestive tumours has not been studied previously. We hypothesised that CTLP is present in GI tract tumour tissue and that it contributes to tumour progression is due its activity against various immunomodulatory proteins critical for the regulation of tumour microenvironment and inflammation.

\section{MATERIALS AND METHODS}

\section{In vivo evaluation of the presence of Td-CTLP in cancer tissues}

Antibody preparation. The CTLP antibody was prepared accordingly (Grenier et al, 1990). The animal experiments were approved by the experimentation ethics committee of Université Laval (license no. 94-190). Briefly, the purified CTLP (15 $\mu \mathrm{g})$ was injected intramuscularly into a New Zealand White rabbit with complete Freund adjuvant. Subsequent intramuscular injections, without adjuvant, were done on days $8,14,22,36$, and 50 . The rabbit was bled via the marginal ear vein on day 57 . The purified immunoglobulin $\mathrm{G}$ (IgG) fraction was prepared by passing the antiserum through a column of protein A-Sepharose CL 4B (Sigma Chemical Co., St Louis, MO, USA). The sample was exhaustively washed on the column with $0.1 \mathrm{~m}$ borate $-0.5 \mathrm{M} \mathrm{NaCl}$ buffer $(\mathrm{pH}$ 8.4). Immunoglobulin $G$ was then eluted with $0.1 \mathrm{M}$ glycine $-0.5 \mathrm{M}$ $\mathrm{NaCl}$ buffer ( $\mathrm{pH}$ 2.5), followed by dialysis against $50 \mathrm{~mm}$ phosphate-buffered saline ( $\mathrm{pH}$ 7.2). The anti-CTLP IgG fraction did not react with Treponema vincentii, an oral spirochete closely related to $T d$, in immunofluorescence and ELISA assays indicating high specificity of the antibody.

Immunohistochemical analysis. A total of 149 orodigestive tumour tissue samples were obtained from the archives of Department of Pathology, Helsinki University Hospital. The study design complied with the Declaration of Helsinki and was approved by the Ethical Committee of Helsinki University Hospital. Tissue samples comprised squamous cell carcinomas (SCCs) of tongue $(n=29)$, tonsil $(n=25)$, oesophagus $(n=3)$, and adenocarcinoma of stomach $(n=32)$, pancreas $(n=6)$, and colon $(n=54)$. All samples were formalin-fixed and paraffin-embedded. Gingival tissue sample from patients with periodontitis verified positive for $T d$ by PCR were included as positive control (Marttila et al, 2014). Mammae ductal carcinoma $(n=1)$, SCC of the lung $(n=1)$, and various types of thyroid tumour tissues $(n=28)$ were used for comparison as non-orodigestive tumour tissues.

Tissue sections were prepared according to standard protocol (Marttila et al, 2014). Antigens were retrieved in citrate buffer using microwaves (MicroMED T T Mega Histoprocessing Labstation; Milestone Srl, Sorisole, Italy). Immunohistochemical stainings were performed using primary rabbit polyclonal antibody (1:1500 dilution) against Td-CTLP. The tissue sections were then incubated with 1:200 dilution of corresponding biotinylated anti-rabbit secondary antibody from the kit (Vectastain Elite ABC kit; Vector Laboratories, Burlingame, CA, USA) and further incubated with avidin-biotin-peroxidase complex. 3-amino-9-ethylcarbazole with $0.03 \%$ hydrogen peroxidase was used to reveal peroxidase-binding site and counterstained with Mayer's haematoxylin. Negative 
control stainings were performed with non-immune speciesspecific Rabbit IgG (Vector Laboratories, Burlingame, CA, USA) and by omitting the primary antibody. The samples were examined and images were taken with Leica DM6000 light microscope connected to a digital camera (DFC420 and DFC365FX; Leica Microsystems, Wetzlar, Germany).

\section{In vitro analysis of $T d$-CTLP activity}

Purification of Td-CTLP and proMMPs. The purification of TdCTLP was done as previously described (Uitto et al, 1988; Grenier et al, 1990). The CTLP was isolated from a sonicated cell extract of Td 35405. The purity of the protease was verified by silver staining and proteolytic activity detection on SDS-PAGE, which showed only a single band with an apparent molecular mass of $95 \mathrm{kDa}$. The protease showed a high specificity for the synthetic chromogenic peptide succinyl-L-alanyl-L-alanyl-L-prolyl-L-phenylalanine- $p$-nitroanilide, was activated by reducing agents, and was inhibited by phenylmethylsulfonyl fluoride and sulfhydryl group reagents, which was consistent with the characteristics previously.

The purification of proMMP-8 and proMMP-9 was done as previously described (Sorsa et al, 1992, 1997). Briefly, supernatant from stimulated human neutrophils was subjected to gel filtration ion-exchange chromatography followed by affinity chromatography on heat-denatured type I collagen (gelatin) coupled to Sepharose for proMMP-9 and Cibacron-Blue Sepharose for proMMP-8. The purified proMMP-8 and proMMP-9 were latent and displayed no enzymatic activities.

Degradation of immunomodulatory components by Td-CTLP. Native soluble type I and type II collagen (for MMP-8 and TdCTLP) as well as gelatin-zymography (for MMP-9 and Td-CTLP) degradation assays were performed as previously described (Sorsa et al, 1992, 1997). Native soluble $1.5 \mu \mathrm{M}$ type I and II collagens were purified and characterised by $\mathrm{CNBr}$-cleavage analysis as previously described (Sorsa et al, 1988). A unit of $400 \mathrm{ng}$ of $75 \mathrm{kDa}$ proMMP$8,400 \mathrm{ng}$ of $92 \mathrm{kDa}$ proMMP-9, $400 \mathrm{ng}$ of $28 \mathrm{kDa}$ human recombinant TIMP-1 (R\&D System, Oxon, UK), $400 \mathrm{ng}$ of $21 \mathrm{kDa}$ human recombinant TIMP-2 (R\&D System), $400 \mathrm{ng} \alpha 1$ AC (Sigma-Aldrich, St Louis, MO, USA), and $400 \mathrm{ng} \mathrm{C1q} \mathrm{(Abcam,}$ Cambridge, UK) were incubated with $80 \mathrm{ng}$ of purified Td-CTLP for various time periods extending from 0 to $60 \mathrm{~min}$ at $37^{\circ} \mathrm{C}$. As positive control, proMMP- 8 and -9 were also incubated for $60 \mathrm{~min}$ $\left(37^{\circ} \mathrm{C}\right)$ with $1 \mathrm{~mm}$ organomercurial activator aminophenylmercuric acetate (APMA; Sigma-Aldrich) and $25 \mathrm{ng}$ human tumourassociated trypsin-2. Human tumour-associated trypsin-2 was purified from serum-free conditioned medium of COLO-205 human colon adenocarcinoma cells (American Type Culture Collection, CCL-222, Rockville, MD, USA) (Sorsa et al, 1997). After the incubations, the samples were removed, boiled for $5 \mathrm{~min}$ in Laemmli's sample buffer and run on SDS-PAGE (Coomassie Brilliant Blue staining) and western immunoblotted to analyse the conversions and fragmentations. Gelatin-zymography and sample preparations for it were performed as described previously (Sorsa et al, 1997).

\section{RESULTS}

Localisation of $T d$-CTLP in orodigestive cancer tissues in vivo. To study the presence of Td-CTLP in malignant tumours, various cancer specimens were stained by immunohistochemistry. Td-CTLP was detected in all types of orodigestive cancer tissues, including SCCs of tongue, tonsil, and oesophagus (Figure $1 \mathrm{~A}-\mathrm{C}$ ). The treponemal proteinase was also detected in adenocarcinomas of the stomach, pancreas, and colon (Figure 1D-F). However, it was not detected in every sample as described by the number of positive samples in Figure 1. Immunoreactivity for Td-CTLP was mainly detected in granular deposits in epithelial cell cytoplasm within tumours. In addition, it was detected in some normal tonsillar epithelial cells. Some mucus secretory cells such as goblet cells, acinar cells, and ductal cells contained Td-CTLP as well. Mononuclear inflammatory cells also showed weak positivity in some specimens of all cancer types (data not shown).

As expected, the staining results on $T d$-positive gingival tissue affected by periodontitis showed positivity for Td-CTLP and negativity in non-orodigestive tumours, including breast and lung cancer tissue (data not shown). For thyroid, limit and weak expression was found in one follicular thyroid adenoma and one follicular thyroid carcinoma, but negative in the rest, including papillary thyroid carcinoma, medullary thyroid carcinoma, and hyperplastic thyroid tissue (data not shown). Negative controls, including stainings with IgG antibody and without primary antibody showed no positivity (data not shown).

Activity of Td-CTLP in vitro. To elucidate mechanisms in which way the Td-CTLP may affect tumour growth, some key proteins connected to the regulation of the proteinase activities of the tumours were incubated in the presence of the Td-CTLP (Figures 2-4). Treponema denticola-CTLP converted time-dependently the $75 \mathrm{kDa}$ proMMP-8 to $60 \mathrm{kDa}$ active forms of MMP- 8 within 20-60 min as revealed by SDS-PAGE analysis (Figure 2A). The conversion of $92 \mathrm{kDa}$ proMMP-9 to $80-82 \mathrm{kDa}$ active form of MMP-9 by Td-CTLP also occurred in 20-60 min (Figure 2B). Corresponding conversions of proMMP- 8 and -9 to their active counterparts were also obtained by $1 \mathrm{~mm}$ APMA and human tumour-associated trypsin-2, used as positive controls (lane 2 in Figure $2 \mathrm{~A}$ and $\mathrm{B}$, respectively). Furthermore, Td-CTLP fragmented $28 \mathrm{kDa}$ TIMP-1 and $21 \mathrm{kDa}$ TIMP-2 to small peptides and lowermolecular-weight fragment as analysed by SDS-PAGE (Figure 2C and $\mathrm{D}$, respectively). The proMMP conversions analysed by SDSPAGE were further confirmed by western immunoblotting (Figure $3 \mathrm{~A}$ and $\mathrm{B})$. Treponema denticola-CTLP also efficiently fragmented $65 \mathrm{kDa} \alpha-1-\mathrm{AC}$ (Figure $4 \mathrm{~A}$ ) and $68 \mathrm{kDa} \mathrm{Clq}$ (Figure $4 \mathrm{~B}$ ) to smaller peptides.

Td-CTLP did not degrade native type I and II collagens (Figure 5A, lanes 4 and 8 , respectively). MMP-8 alone degraded to some extent type I and II collagens (Figure 5A, lanes 3 and 6, respectively). MMP-8 treated by Td-CTLP for $60 \mathrm{~min}$ prior incubation with type I and II collagen resulted to significantly enhanced degradation of both these collagens, preferably type II collagen (Figure 5A, lanes 3 and 7, respectively). Type I gelatinzymographic analysis revealed that Td-CTLP is a gelatinolytic 80 $95 \mathrm{kDa}$ protease (Figure 5B, lane 1) and time-dependently converted gelatinolytic $92 \mathrm{kDa}$ pro MMP-9 (Figure 5B, lane 2) to active $82 \mathrm{kDa}$ form (Figure 5B, lanes 3-5, indicated by an arrow).

\section{DISCUSSION}

In this study, we showed for the first time that the CTLP, a key virulence factor specific to the oral spirochete Treponema denticola $(T d)$, is present in several orodigestive tumours. In addition, our in vitro experiments provide evidence that Td-CTLP shows immunomodulatory activity that can have a crucial role in promoting and regulating carcinogenesis. $T d$ is known to be a key pathogen in periodontitis, a chronic inflammatory disease of the tooth supporting connective tissue caused by the dysbiotic microbiome. Previous studies regarding Td-CTLP have focused on its potential role in the pathogenesis of periodontitis. However, the role of the treponemal proteinase in carcinogenesis has not been considered before. Recent studies have suggested a link between periodontitis and oral cancer (Gholizadeh et al, 2016). The current knowledge on the role of $T d$ in chronic inflammation, and its 

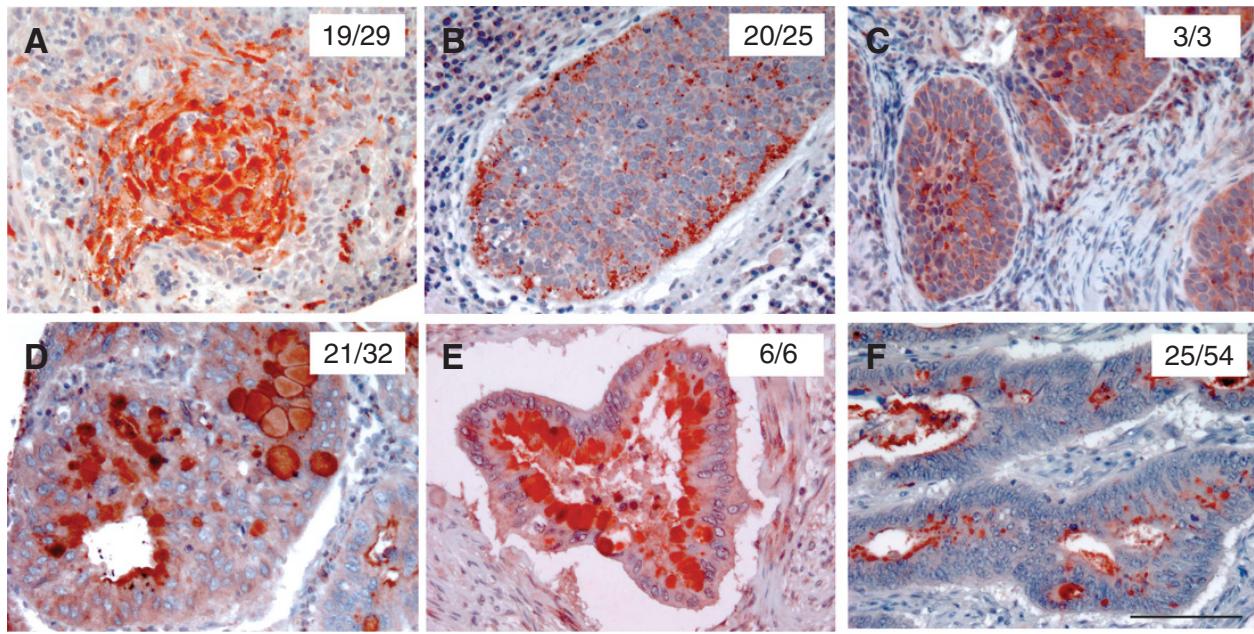

Figure 1. The presence of Td-CTLP in orodigestive tumour tissues. Immunohistochemical stainings of the Td-CTLP in tongue (A), tonsillar (B), oesophageal (C), gastric (D), pancreatic $(\mathbf{E})$, and colon cancer $(\mathbf{F})$ tissues. Number of positive tissue samples per total sample size for each tumour type shown. 3-amino-9-ethylcarbazole (AEC) was used as chromogen (red). All red-stained areas on each tissue section indicate specific detection of Td-CTLP. Scale bar is $100 \mu \mathrm{m}$, relevant for all panels.

highly invasive and immunomodulatory nature via its powerful proteolytic machinery, urged us to investigate whether this spirochete with the aid of its proteolytic enzyme has a part in the aetiology of cancer.

Currently, there is very limited data available about the presence of $T d$ in malignant tumours. Narikiyo et al, using culture independent-methods, reported frequent presence of $T d$ together with oral streptococci within oesophageal tumours obtained from several centres globally. Td was more frequently isolated from tumour than non-tumour sites. In our study, Td-CTLP was also present in oesophageal tumour samples, and interestingly in the majority of other orodigestive tumours as well.

Td can be isolated from the oral cavity of healthy individuals, but in disease, the number of $T d$ has been shown to increase substantially alongside other periodontal pathogens (Dashper et al, 2011). The presence of Td-CTLP within orodigestive tumours implicates that mucosal exposure along the GI tract to $T d$ is due to ingestion of the spirochete with food and saliva. In our study, this implication is partly contradicted by the discovery of Td-CTLP in pancreatic cancer. This can be explained by the fact that the pancreas is connected to the bowel by its duct. It is also possible that bacteria reach the pancreas via blood circulation. To accompany the latter, $T d$ has been detected in carotid and aortic atheromatous plaques in patients with chronic periodontitis (Cavrini et al, 2005).

We have previously investigated the localisation of $T d$ and its CTLP in oral gingival tissues affected by periodontitis (Marttila et al, 2014). The localisation of Td-CTLP correlated well with the presence of $T d$ bacteria, and the CTLP was detected in all layers from mucosal biofilm all the way through the epithelium to the connective tissue, thus indicating the invasiveness of this organism. Similar to our previous study, Td-CTLP was mainly localised intracellularly within tumour cells in the current study. Td-CTLP is known to facilitate binding of the spirochete to other bacterial counterparts in polymicrobial biofilms and $\mathrm{Td}$ has been found to coincide in the tissues with $P g$, a periodontal pathogen that has been associated with various types of orodigestive malignancies such as colorectal, pancreatic, and oral cancers (Nagy et al, 1998; Ahn et al, 2012a; Michaud et al, 2013).

In cancer progression and proliferation proteolytic activity is a crucial factor, mainly carried out by MMPs. In vitro, Td-CTLP converted proMMP- 8 and -9 to their active forms as demonstrated by western blots and type I and II collagen and gelatin- zymographic MMP-activity assays. In fact, the Td-CTLP-activated MMP-8 degraded preferentially native soluble type II collagen relative to type I collagen, which is according to recorded substrate specificity of MMP-8 (Hasty et al, 1987; Sorsa et al, 1988). The MMPs regulate tumour cell tissue invasion and extravasation, angiogenesis and inflammation, and thus have a major impact on tumour microenvironment (Kessenbrock et al, 2010). Elevated levels of MMP-9 have been associated with poor prognosis and metastasis in tongue, oesophageal, gastric, pancreatic, and colorectal cancers (Zeng et al, 2013; Aparna et al, 2015; Jakubowska et al, 2016). In periodontitis, the presence of $T d$ is associated with increased levels of MMP-8 and -9 (Yakob et al, 2013). Td is known to trigger MMP-8 and - 9 expression and secretion by inflammatory cells in vitro (Ding et al, 1996; Choi et al, 2003). Interestingly, there has been controversy over the effect of MMP- 8 in cancer. Matrix metalloproteinase- 8 is overexpressed in oropharyngeal cancers and moreover its expression is associated with poor prognosis in ovarian and bladder cancer (Moilanen et al, 2002; Stadlmann et al, 2003; Kader et al, 2006). In addition, MMP-8 overexpression also exhibits tumour- and metastasis-suppressing roles and associates with improved survival in tongue and breast cancers (GutierrezFernandez et al, 2008; Korpi et al, 2008). In addition to ECM modulation, MMP-8 and -9 show potent immunomodulatory activity in tumour tissues (Kessenbrock et al, 2010).

Tissue inhibitors of MMPs act on tumour status primarily through inhibition of MMPs. In addition, MMP-independent effects of TIMPs on cell growth and differentiation, cell migration, anti-angiogenesis, anti- and pro-apoptosis, and synaptic plasticity have been reported (Brew \& Nagase, 2010). TIMP-1 and -2 are widely expressed (Jackson et al, 2016). TIMP-1 can promote tumour progression, angiogenesis, inhibit apoptosis, and it is associated with poor survival. TIMP-2 is primarily known for its inhibitory activity on MMP-2, but it also has MMP-independent activities. In the current study, TIMP- 1 and -2 were degraded by $T d$-CTLP to inactive forms. Thus, by acting on TIMPs, Td-CTLP could exert effect on MMP activity and inhibit MMP-independent activities of TIMPs within the tumour site contributing carcinogenesis.

In our study, we examined two other inflammation related factors, acute phase protein $\alpha-1-\mathrm{AC}$ and complement component C1q. Td-CTLP degraded both proteins. Expression of $\alpha$-1-AC has been reported in pancreatic and gastric cancers (Allgayer et al, 1998; Koomen et al, 2005). A recent report shows that $\mathrm{Clq}$ is 
A

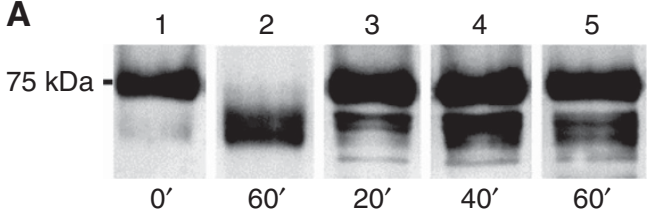

B
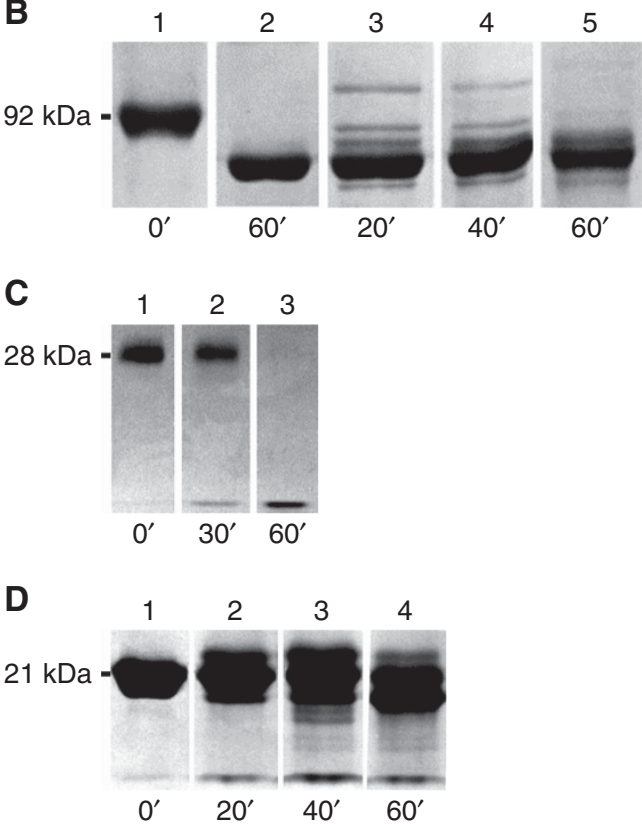

Figure 2. SDS-PAGE of fragmentation of pro MMP-8 and -9 and TIMP1 and -2 by Td-CTLP. (A) Lane 1: proMMP-8 incubated with buffer; lane 2: proMMP-8 + APMA (organomercurial MMP-8 activator) indicates that proMMP-8 was completely activated by APMA after 60 min; lanes 3-5: Td-CTLP activated proMMP-8 during 20, 40, and 60 min incubations. (B) Lane 1: proMMP-9 incubated with buffer; lane 2: proMMP9 + APMA (organomercurial MMP-8 activator) indicates that proMMP-9 was completely activated by APMA after $60 \mathrm{~min}$; lanes 3-5: Td-CTLP activated proMMP-9 during 20, 40, and 60 min incubations. (C) Lane 1: TIMP-1 incubated with buffer; lanes 2 and 3: TIMP-1 incubated with Td-CTLP indicates that TIMP-1 was degraded to smaller fragments after $60 \mathrm{~min}$. (D) Lane 1: TIMP-2 incubated with buffer; lanes 2-4: TIMP-2 incubated with $T d$-CTLP indicates that TIMP-2 was degraded to smaller fragments after $60 \mathrm{~min}$.

present in GI cancers and that locally synthesised C1q can promote cancer cell adhesion, migration, and proliferation in vivo (Bulla et al, 2016).

The strength of the study is the use of a highly specific polyclonal antibody against Td-CTLP. In vivo localisation of the enzyme in various orodigestive tumours provide a potential insight into its biological effects. The lack of functional analysis of CTLP in tumours is a limitation of the study. In addition, the proteins investigated in vitro were not stained alongside CTLP in tumour sites, but as discussed here plentiful of reports highlight their presence and role within orodigestive tumours. The enzymesubstrate ratios used in vitro were based on our previous studies with microbial proteases and MMPs, and should not be regarded to be physiologically or pathologically relevant (Uitto et al, 1988; Sorsa et al, 1992). There are no data available regarding the level of such microbial proteases in human body fluids and tissues, and therefore these ratios are practically impossible to estimate. These data thus represent a potential existence of such microbialprotease-MMP tissue destructive cascade.

Our data open a new perspective into the possible role of periodontal pathogens as aetiologic factors in orodigestive
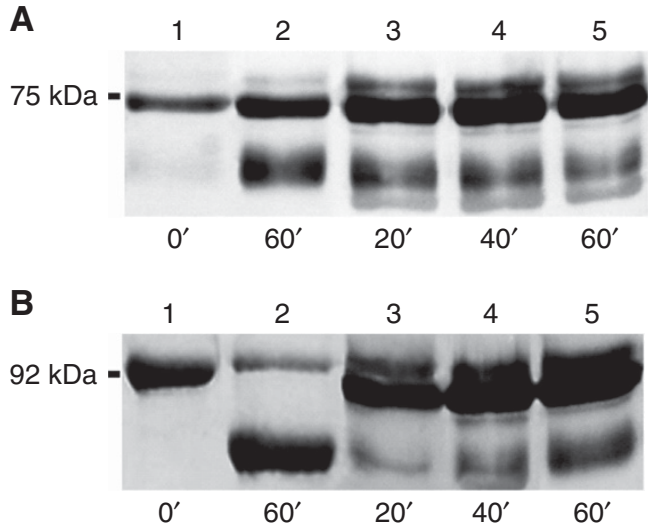

Figure 3. Western immunoblot analysis of conversion of pro MMP-8 and -9 by Td-CTLP. (A) Lane 1: pro MMP-8 incubated with buffer; lane 2: pro MMP-8 incubated with tumour-associated trypsin-2; lanes 3-5: pro MMP-8 incubated with Td-CTLP for 20, 40, and 60 min. (B) Lane 1: pro MMP-9 incubated with buffer; lane 2: pro MMP-9 incubated with tumour-associated trypsin-2; lanes 3-5: pro MMP-9 incubated with TdCTLP for 20, 40, and $60 \mathrm{~min}$, respectively. Treponema denticola-CTLP and tumour-associated trypsin-2 converted pro MMP-8 and -9 to their active forms.
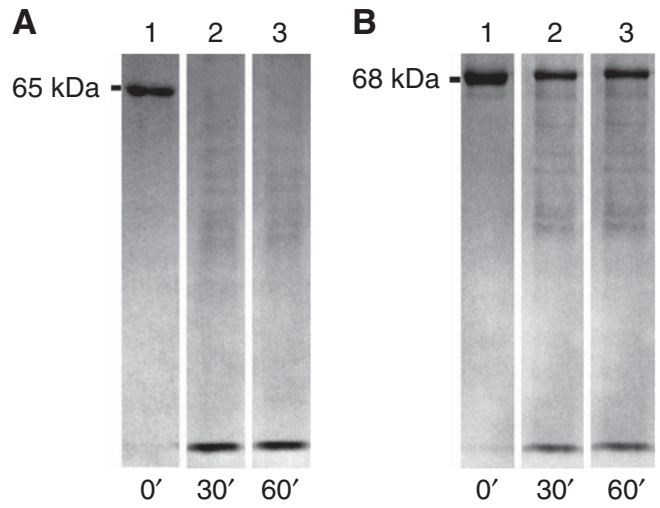

Figure 4. SDS-PAGE of fragmentation of immunomodulatory proteins by Td-CTLP. (A) Lane 1: $\alpha-1-A C$ incubated with buffer; lanes 2 and 3: $\alpha-1-A C$ incubated with Td-CTLP indicated that Td-CTLP completely degraded $\alpha-1-A C$ in 30 and $60 \mathrm{~min}$. (B) Lane 1: C1q incubated with buffer; lanes 2 and 3: C1q incubated with Td-CTLP indicated that Td-CTLP degraded C1q in 30 and 60 min incubations.

carcinogenesis. Pathogenic oral bacteria may indeed be translocated into the GI tumours and modulate their growth. Further studies are warranted to provide deeper understanding about the mechanisms of $T d$ and its virulence factor CTLP in carcinogenesis.

\section{ACKNOWLEDGEMENTS}

This study was supported by Directorate General of Human Resource for Science, Technology and Higher Education of Indonesia, Center for International Mobility (CIMO TM-159588), Selma and Maja-Lisa Selanders fund, Otto A Malm Foundation, FINDOS travel grant, Sigrid Jusélius Foundation, Finska Läkaresällskapet, FONDECYT 1160741 grant, Helsinki University Hospital Research Foundation (TYH 2016251, TYH 2017251, and Y 1149SUL32), Helsinki, Finland, and the Karolinska Institutet, Stockholm, Sweden. Dr Anne Järvensivu, DDS is acknowledged for providing the periodontitis-affected human tissue specimen. 

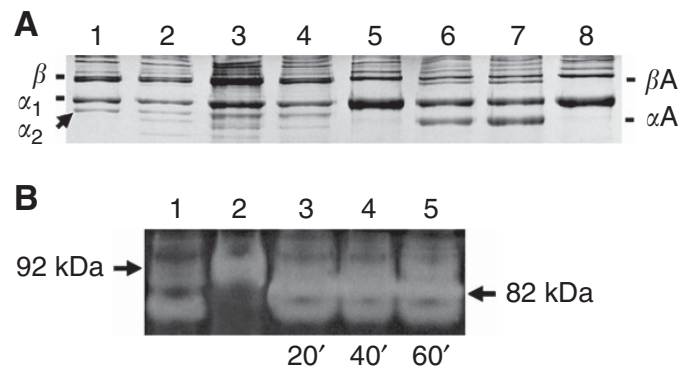

Figure 5. (A) SDS-PAGE (10\%; Coomassie Brilliant Blue staining) analysis of the effects of Td-CTLP on native soluble type I and II collagen degradation by MMP-8. Lane 1: $1.5 \mu \mathrm{M}$ type I collagen; lane 2: type I collagen incubated with pro MMP-8; lane 3: type I collagen incubated with Td-CTLP-treated MMP-8; lane 4: type I collagen incubated with Td-CTLP; lane 5: type II collagen; lane 6: type II collagen incubated with MMP-8; lane 7: type II collagen incubated with Td-CTLP. $\alpha_{1}, \alpha_{2}$, and $\beta_{1}$ indicate intact soluble native type I and II collagen monomers and dimers and $\beta \mathrm{A}$ and $\alpha \mathrm{A}$ indicate their characteristic $\frac{3}{4}$-cleavage products resulting from collagenase activity. (B) Type I gelatin-zymographic analysis of the effects of Td-CTLP on human $92 \mathrm{kDa}$ proMMP-9. Gelatinolytic 80-95 kDa Td-CTLP (lane 1) converts time-dependently (0-60 min) $92 \mathrm{kDa}$ pro MMP-9 (lane 2) to $82 \mathrm{kDa}$ gelatinolytically active form of MMP-9 (lanes $3-5$, indicated by an arrow).

\section{CONFLICT OF INTEREST}

The authors declare no conflict of interest.

\section{REFERENCES}

Ahn J, Chen CY, Hayes RB (2012a) Oral microbiome and oral and gastrointestinal cancer risk. Cancer Causes Control 23: 399-404.

Ahn J, Segers S, Hayes RB (2012b) Periodontal disease, Porphyromonas gingivalis serum antibody levels and orodigestive cancer mortality. Carcinogenesis 33: 1055-1058.

Allgayer H, Babic R, Grützner KU, Beyer BC, Tarabichi A, Schildberg FW, Heiss MM (1998) Tumor-associated proteases and inhibitors in gastric cancer: analysis of prognostic impact and individual risk protease patterns. Clin Exp Metastasis 16: 62-73.

Aparna M, Rao L, Kunhikatta V, Radhakrishnan R (2015) The role of MMP-2 and MMP-9 as prognostic markers in the early stages of tongue squamous cell carcinoma. J Oral Pathol Med 44: 345-352.

Brew K, Nagase H (2010) The tissue inhibitors of metalloproteinases (TIMPs): an ancient family with structural and functional diversity. Biochim Biophys Acta 1803: 55-71.

Bulla R, Tripodo C, Rami D, Ling GS, Agostinis C, Guarnotta C, Zorzet S, Durigutto P, Botto M, Tedesco F (2016) C1q acts in the tumour microenvironment as a cancer-promoting factor independently of complement activation. Nat Commun 7: 10346.

Cavrini F, Sambri V, Moter A, Servidio D, Marangoni A, Montebugnoli L, Foschi F, Prati C, Bartolomeo R, Di, Cevenini R (2005) Case report molecular detection of Treponema denticola and Porphyromonas gingivalis in carotid and aortic atheromatous plaques by FISH: report of two cases. J Med Microbiol 54: 93-96.

Choi B-K, Lee HJ, Kang JH, Jeong GJ, Min CK, Yoo Y-J (2003) Induction of osteoclastogenesis and matrix metalloproteinase expression by the lipooligosaccharide of Treponema denticola. Infect Immun 71: 226-233.

Cogoni V, Morgan-smith A, Fenno JC, Jenkinson HF, Dymock D (2012) Treponema denticola chymotrypsin-like proteinase ( CTLP ) integrates spirochaetes within oral microbial communities. Microbiology 158: 759-770.

Dashper SG, Seers C a, Tan KH, Reynolds EC (2011) Virulence factors of the oral spirochete Treponema denticola. J Dent Res 90: 691-703.

Ding Y, Uitto VJ, Haapasalo M, Lounatmaa K, Konttinen YT, Salo T, Grenier D, Sorsa T (1996) Membrane components of Treponema denticola trigger proteinase release from human polymorphonuclear leukocytes. J Dent Res 75: 1986-1993.

Fenno JC (2012) Treponema denticola interactions with host proteins. J Oral Microbiol 4: 9929.

Fitzpatrick SG, Katz J (2010) The association between periodontal disease and cancer: a review of the literature. J Dent 38: 83-95.

Gallimidi AB, Fischman S, Revach B, Bulvik R, Rubinstein AM, Nussbaum G, Elkin M (2015) Periodontal pathogens Porphyromonas gingivalis and Fusobacterium nucleatum promote tumor progression in an oral-specific chemical carcinogenesis model. Oncotarget 6: 22613-22623.

Gholizadeh P, Eslami H, Yousefi M, Asgharzadeh M, Aghazadeh M, Kafil HS (2016) Role of oral microbiome on oral cancers, a review. Biomed Pharmacother 84: 552-558.

Grenier D, Uitto VJ, McBride B (1990) Cellular location of a Treponema denticola chymotrypsinlike protease and importance of the protease in migration through the basement membrane. Infect Immun $\mathbf{5 8}$ : 347-351.

Groeger S, Domann E, Gonzales JR, Chakraborty T, Meyle J (2011) B7-H1 and B7-DC receptors of oral squamous carcinoma cells are upregulated by Porphyromonas gingivalis. Immunobiology 216: 1302-1310.

Gutierrez-Fernandez A, Fueyo A, Folgueras AR, Garabaya C, Pennington CJ, Pilgrim S, Edwards DR, Holliday DL, Jones JL, Span PN, FCGJ Sweep, Puente XS, Lopez-Otin C (2008) Matrix metalloproteinase-8 functions as a metastasis suppressor through modulation of tumor cell adhesion and invasion. Cancer Res 68: 2755-2763.

Hasty K A, Jeffrey JJ, Hibbs MS, Welgus HG (1987) The collagen substrate specificity of human neutrophil collagenase. J Biol Chem 262: 10048-10052.

Jackson HW, Defamie V, Waterhouse P, Khokha R (2016) TIMPs: versatile extracellular regulators in cancer. Nat Rev Cancer 17: 38-53.

Jakubowska K, Pryczynicz A, Januszewska J, Sidorkiewicz I, Kemona A, Niewiński A, Lewczuk Ł, Kędra B, Guzińska-Ustymowicz K (2016) Expressions of matrix metalloproteinases 2, 7, and 9 in carcinogenesis of pancreatic ductal adenocarcinoma. Dis Markers 2016: 1-7.

Jiang Y, Goldberg ID, Shi YE (2002) Complex roles of tissue inhibitors of metalloproteinases in cancer. Oncogene 21: 2245-2252.

Kader AK, Shao L, Dinney CP, Schabath MB, Wang Y, Liu J, Gu J, Grossman HB, Wu X (2006) Matrix metalloproteinase polymorphisms and bladder cancer risk. Cancer Res 66: 11644-11648.

Karashima S, Kataoka H, Itoh H, Maruyama R, Koono M (1990) Prognostic significance of alpha-1-antitrypsin in early stage of colorectal carcinomas. Int J Cancer 45: 244-250.

Kessenbrock K, Plaks V, Werb Z (2010) Matrix metalloproteinases: regulators of the tumor microenvironment. Cell 141: 52-67.

Kittas C, Aroni K, Matani A, Papadimitriou CS (1982) Immunocytochemical demonstration of a1-antitrypsin and a1-antichymotrypsin in human gastrointestinal tract. Hepatogastroenterology 29: 275-277.

Koomen JM, Shih LN, Coombes KR, Li D, Xiao L, Fidler IJ, Abbruzzese JL, Kobayashi R (2005) Plasma protein profiling for diagnosis of pancreatic cancer reveals the presence of host response proteins. Clin Cancer Res 11: $1110-1118$

Korpi J, Kervinen V, Mäklin H, Väänänen A, Lahtinen M, Läärä E, Ristimäki A Thomas G, Ylipalosaari M, Strö M PÅ, Lopez-Otin C, Sorsa T, Kantola S, Pirilä E, Salo T (2008) Collagenase-2 (matrix metalloproteinase-8) plays a protective role in tongue cancer. Br J Cancer 98: 766-775.

Marttila E, Järvensivu A, Sorsa T, Grenier D, Richardson M, Kari K, Tervahartiala T, Rautemaa R (2014) Intracellular localization of Treponema denticola chymotrypsin-like proteinase in chronic periodontitis. J Oral Microbiol 6: 1-6.

Michaud DS (2013) Role of bacterial infections in pancreatic cancer. Carcinogenesis 34: 2193-2197.

Michaud DS, Izard J, Wilhelm-Benartzi CS, You D-H, Grote VA, Tjønneland A, Dahm CC, Overvad K, Jenab M, Fedirko V, Boutron-Ruault MC, Clavel-Chapelon F, Racine A, Kaaks R, Boeing H, Foerster J, Trichopoulou A, Lagiou P, Trichopoulos D, Sacerdote C, Sieri S, Palli D, Tumino R, Panico S, Siersema PD, Peeters PH, Lund E, Barricarte A, Huerta J-M, Molina-Montes E, Dorronsoro M, Quirós JR, Duell EJ, Ye W, Sund M, Lindkvist B, Johansen D, Khaw K-T, Wareham N, Travis RC, Vineis $\mathrm{P}$, Bueno-de-Mesquita HB, Riboli E (2013) Plasma antibodies to oral bacteria and risk of pancreatic cancer in a large European prospective cohort study. Gut 62: 1764-1770.

Moilanen M, Pirilä E, Grénman R, Sorsa T, Salo T (2002) Expression and regulation of collagenase-2 (MMP-8) in head and neck squamous cell carcinomas. J Pathol 197: 72-81. 
Nagy KN, Sonkodi I, Szöke I, Nagy E, Newman HN (1998) The microflora associated with human oral carcinomas. Oral Oncol 34: 304-308.

Narikiyo M, Tanabe C, Yamada Y, Igaki H, Tachimori Y, Kato H, Montesano R, Sakamoto H, Nakajima Y, Sasaki H (2004) Frequent and preferential infection of Treponema denticola, Streptococcus mitis, and Streptococcus anginosus in esophageal cancers. Cancer Sci 95: 569-574.

Parkin DM (2006) The global health burden of infection-associated cancers in the year 2002. Int J Cancer 3044: 3030-3044.

Ribet D, Cossart P (2015) How bacterial pathogens colonize their hosts and invade deeper tissues. Microbes Infect 17: 173-183.

Rubinstein MR, Wang X, Liu W, Hao Y, Cai G, Han YW (2013) Fusobacterium nucleatum promotes colorectal carcinogenesis by modulating E-cadherin/ $\beta$-catenin signaling via its FadA adhesin. Cell Host Microbe 14: 195-206.

Salminen A, Åström P, Metso J, Soliymani R, Salo T, Jauhiainen M, Pussinen PJ, Sorsa T (2015) Matrix metalloproteinase 8 degrades apolipoprotein A-I and reduces its cholesterol efflux capacity. FASEB J 29: 1435-1445.

Sorsa T, Uitto VJ, Suomalainen K, Vauhkonen M, Lindy S (1988) Comparison of interstitial collagenases from human gingiva, sulcular fluid and polymorphonuclear leukocytes. J Periodontal Res 23: 386-393.

Sorsa T, Ingman T, Suomalainen K, Haapasalo M, Konttinen YT, Lindy O, Saari H, Uitto VJ (1992) Identification of proteases from periodontopathogenic bacteria as activators of latent human neutrophil and fibroblast-type interstitial collagenases. Infect Immun $\mathbf{6 0}$ : 4491-4495.

Sorsa T, Salo T, Koivunen E, Tyynela J, Konttinen YT, Bergmann U, Tuuttila A, Niemi E, Teronen O, Heikkila P, Tschesche H, Leinonen J, Osman S, Stenman U-H (1997) Activation of type IV procollagenases by human tumor-associated trypsin-2. J Biol Chem 272: 21067-21074.
Stadlmann S, Pollheimer J, Moser PL, Raggi A, Amberger A, Margreiter R, Offner FA, Mikuz G, Dirnhofer S, Moch H (2003) Cytokine-regulated expression of collagenase-2 (MMP-8) is involved in the progression of ovarian cancer. Eur J Cancer 39: 2499-2505.

Sun Z, Yang P (2004) Role of imbalance between neutrophil elastase and $\alpha 1$-antitrypsin in cancer development and progression. Lancet Oncol 5: 182-190.

Uitto VJ, Grenier D, Chan ECS, McBride BC (1988) Isolation of a chymotrypsinlike enzyme from Treponema denticola. Infect Immun 56: 2717-2722.

Uitto VJ, Pan YM, Leung WK, Larjava H, Ellen RP, Finlay BB, McBride BC (1995) Cytopathic effects of Treponema denticola chymotrypsin-like proteinase on migrating and stratified epithelial cells. Infect Immun 63: 3401-3410.

Verma S, Kesh K, Ganguly N, Jana S, Swarnakar S (2014) Matrix metalloproteinases and gastrointestinal cancers: impacts of dietary antioxidants. World J Biol Chem 5: 355.

Yakob M, Meurman JH, Sorsa T, Söder B (2013) Treponema denticola associates with increased levels of MMP-8 and MMP-9 in gingival crevicular fluid. Oral Dis 19: 694-701.

Zeng R, Duan L, Kong Y, Liang Y, Wu X, Wei X, Yang K (2013) Clinicopathological and prognostic role of MMP-9 in esophageal squamous cell carcinoma: a meta-analysis. Chin J Cancer Res 25: 637-645.

This work is published under the standard license to publish agreement. After 12 months the work will become freely available and the license terms will switch to a Creative Commons AttributionNonCommercial-Share Alike 4.0 Unported License. 